\section{How Do I Visualize A Thin Gold Film On A Smooth Surface}

Mary Mager, University of British Columbia mager@interchange.ubc.ca

A question on the Microscopy Listserver concerned using an FESEM to examine a thin, thermally-evaporated gold film on single crystal germanium. It is always a difficult problem to find small surface details or textures on a smooth surface in the SEM. A surface without visible topography looks the same whether it is in or out of focus, and so the method to make sure the surface is in focus is a slow, iterative one.

My researchers like to give me a one inch square of highly polished steel or titanium and ask me to find and analyze the five tiny inclusions somewhere in the middle. If there is a particular feature or area that I need to examine, I find that area by eye or under a low power, binocular light microscope and mark it with a fine, red felt-tip pen. I use the "fine" or "superfine" red, permanent Lumocolor pens, but others will probably work. A micro-hardness indenter is also useful to mark small features, then surround the area with a red circle. I find that the red ink is visible under the SEM, but the black is not. If it is a small area I draw a circle around it, then a line from the circle to one edge or corner. This reduces the time and effort required to search all over a sample that has been magnified to football-field size: find the line and follow it to the circle. The red pen mark serves two purposes: it limits the search area and it provides a large feature for the initial focus. The mark washes off the sample easily with lab alcohol (Figure 1). Other ways to mark a surface to give a focusing aid are to scratch the surface, if allowed, or to apply something to the surface. Years ago I was given some fine aluminum flake, such as that used to make makeup shimmer, and a tiny dab of that in a corner was an excellent way to find the initial focus. I do not know, however, where to get more.

Start with the usual conditions for low magnification imaging: standard working distance, middle condenser current or spot size and mid-sized aperture. Focus on the edge of the pen mark or scratch. Now shorten the working distance to the

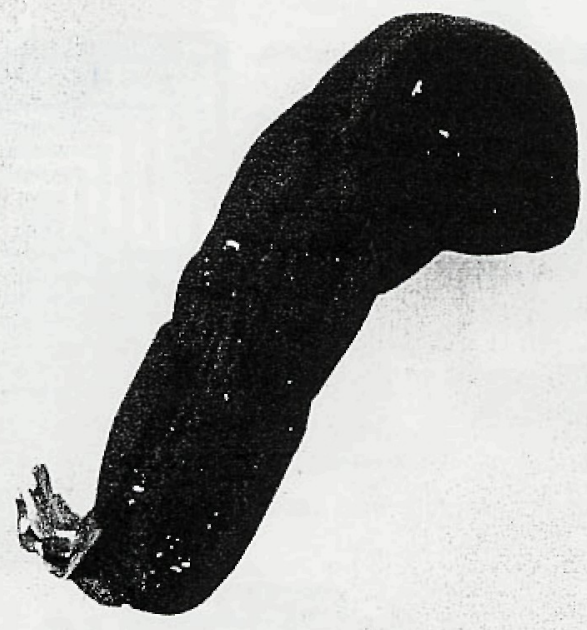

$500 \mu \mathrm{m}$

Figure 1. Low magnification of a polished silicon wafer with a red, felt pen mark. optimum for high resolution, using the edge of the mark to continually find the focus as the $Z$ control is moved. When the highresolution working distance is reached, go to higher magnification and insert a smaller aperture, if your aperture is adjustable. Center the aperture and correct any astigmatism. About this time I start looking off to the side of the mark for a tiny dust mark or surface flaw. Use a slow scan speed or capture a slow-scan digital image and find a little mark with good contrast (Figure 2). I have seldom found a sample so clean that a little bit of something small cannot be found to focus on, but if there isn't anything, then use the edge of the pen mark. Use a slow scan speed or the reduced-area raster to make the snowy image a little clearer. Adjust the condenser or spot size for high resolution, making the spot as small as possible without losing too much contrast (Figure 3 ).

Now I usually go to ridiculously high magnification, two or three times the magnification of the final image, on the edge of this particle. Use manual brightness and contrast to bring up the edges
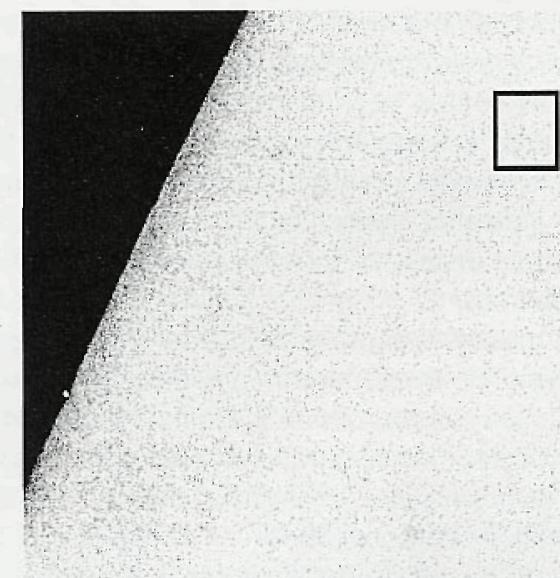

Figure 2. Higher magnification of felt pen mark and small particles.

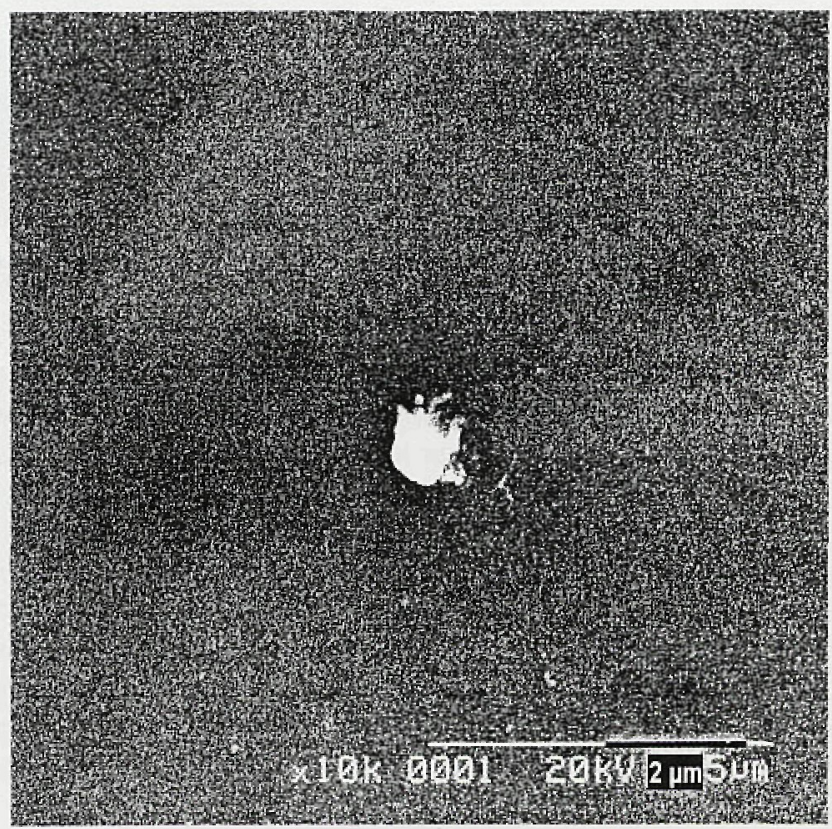

Figure 3. Small particle for fine focussing. 
and carefully focus, align the aperture and correct the astigmatism. Now go to the desired magnification for the image and use the image shift to move this bright particle outside the field of view and re-adjust the contrast and brightness for this smooth surface. The contrast will likely need to be turned up and the brightness down, although the auto brightness and contrast might work. Take a slow or photo speed image of the surface and surface details that not seen on the microscope CRT should become visible (Figures 4 and 5).

On my ten-year-old Hitachi SEM with a tungsten filament, I was able to resolve the gold sputtered coating (see Photo 4), but not the thermal coating. However, I didn't have much gold in the evaporator basket, so perhaps a thicker coating would have shown more features.

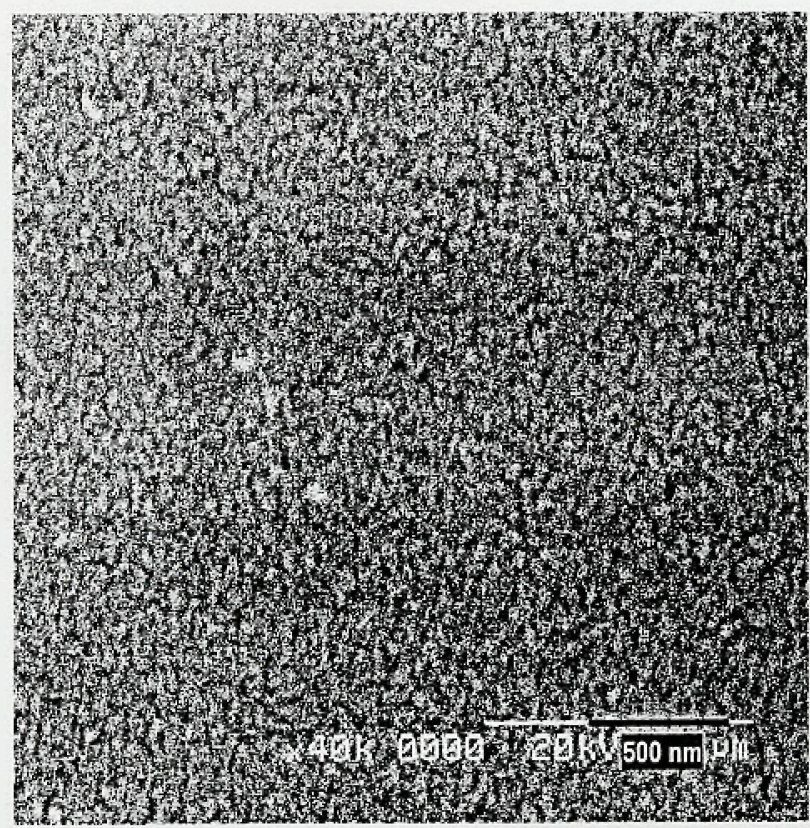

Figure 4. Gold coating on silicon wafer.

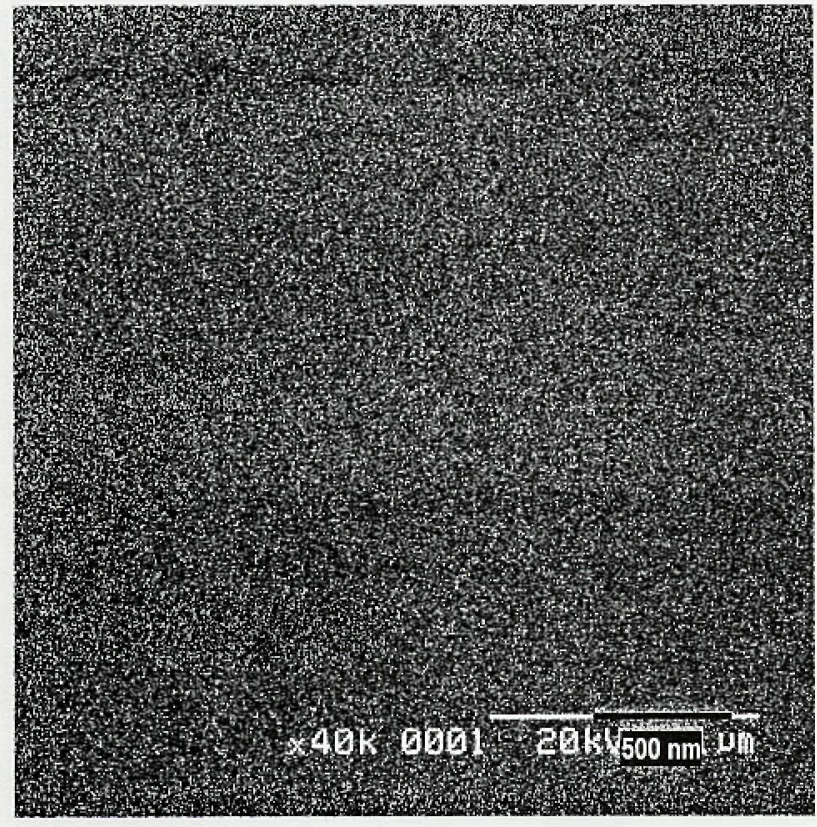

Figure 5. Silicon wafer without gold coating.

\section{STOP HASSLING WTH \\ MULTIPLE SERVICE CONTRACTS!}

START by putting all of your instruments under one service contract with MAS (regardless of make or model). Our expert EM SERVICE GROUP has the knowledge and skills to keep your instrument working at its best.

\begin{tabular}{|c|c|c|c|}
\hline \multicolumn{2}{|c|}{ IEIS / SEM'S } & PREP ECUIPMENT & SPECIAL SERVIGES \\
\hline HITACHI & TOPCON & VACUUM COATERS & STAGES \\
JEOL & ISI & PLASMA ASHERS & BEAM BLANKERS \\
AMRAY CAMBRIDGE & SPUTTER COATERS & CUSTOM DEVICES \\
\hline
\end{tabular}

\section{NEW NEW NEW}

Authorized service representatives for Gatan preparation equipment and Topcon TEM'S.
Contracts and On-Demand Emergency Service at Reasonable Rates from Factory Trained Specialists.

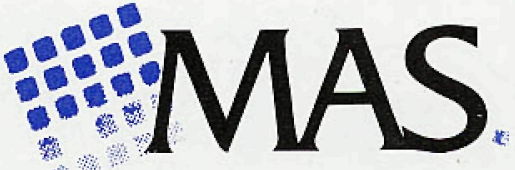

\section{0-421-8451}

3945 Lakefield Court Suwanee, Georgia $30024 \quad 770-866-3200 \quad$ FAX 770-866-3259 616 Hutton Street Suite 101 Raleigh, North Carolina 27606 919-829-7041 FAX919-829-5518 ADVANCED ANALYTICAL PRODUCTS AND SERVICES 